\title{
A TRIPLE OF HEAVY SUBGRAPHS ENSURING PANCYCLICITY OF 2-CONNECTED GRAPHS
}

\author{
WOJCIECH WIDEE \\ Faculty of Applied Mathematics \\ Department of Discrete Mathematics \\ AGH University of Science and Technology \\ al. Mickiewicza 30, 30-059 Krakow, Poland \\ e-mail: widel@agh.edu.pl
}

\begin{abstract}
A graph $G$ on $n$ vertices is said to be pancyclic if it contains cycles of all lengths $k$ for $k \in\{3, \ldots, n\}$. A vertex $v \in V(G)$ is called super-heavy if the number of its neighbours in $G$ is at least $(n+1) / 2$. For a given graph $H$ we say that $G$ is $H$ - $f_{1}$-heavy if for every induced subgraph $K$ of $G$ isomorphic to $H$ and every two vertices $u, v \in V(K), d_{K}(u, v)=2$ implies that at least one of them is super-heavy. For a family of graphs $\mathcal{H}$ we say that $G$ is $\mathcal{H}$ - $f_{1}$-heavy, if $G$ is $H$ - $f_{1}$-heavy for every graph $H \in \mathcal{H}$.

Let $D$ denote the deer, a graph consisting of a triangle with two disjoint paths $P_{3}$ adjoined to two of its vertices. In this paper we prove that every 2-connected $\left\{K_{1,3}, P_{7}, D\right\}$ - $f_{1}$-heavy graph on $n \geq 14$ vertices is pancyclic. This result extends the previous work by Faudree, Ryjáček and Schiermeyer. Keywords: cycle, Fan-type heavy subgraph, Hamilton cycle, pancyclicity.
\end{abstract}

2010 Mathematics Subject Classification: 05C38, 05C45.

\section{REFERENCES}

[1] P. Bedrossian, Forbidden Subgraph and Minimum Degree Conditions for Hamiltonicity (PhD Thesis, Memphis State University, USA, 1991).

[2] P. Bedrossian, G. Chen and R.H. Schelp, A generalization of Fan's condition for Hamiltonicity, pancyclicity and Hamiltonian connectedness, Discrete Math. 115 (1993) 39-59.

doi:10.1016/0012-365X(93)90476-A

[3] A. Benhocine and A.P. Wojda, The Geng-Hua Fan conditions for pancyclic or Hamilton-connected graphs, J. Combin. Theory Ser. B 58 (1987) 167-180. doi:10.1016/0095-8956(87)90038-4 
[4] J.A. Bondy, Pancyclic graphs I, J. Combin. Theory Ser. B 11 (1971) 80-84. doi:10.1016/0095-8956(71)90016-5

[5] J.A. Bondy and U.S.R. Murty, Graph Theory with Applications (Macmillan London and Elsevier, 1976). doi:10.1007/978-1-349-03521-2

[6] G. Chen, B. Wei and X. Zhang, Degree light-free graphs and Hamiltonian cycles, Graphs Combin. 17 (2001) 409-434. doi:10.1007/s003730170018

[7] R. Faudree, Z. Ryjáček and I. Schiermeyer, Forbidden subgraphs and cycle extendability, J. Combin. Math. Combin. Comput. 19 (1995) 109-128.

[8] M. Ferrara, M.S. Jacobson and A. Harris, Cycle lengths in Hamiltonian graphs with a pair of vertices having large degree sum, Graphs Combin. 26 (2010) 215-223. doi:10.1007/s00373-010-0915-z

[9] B. Ning, Fan-type degree condition restricted to triples of induced subgraphs ensuring Hamiltonicity, Inform. Process. Lett. 113 (2013) 823-826. doi:10.1016/j.ipl.2013.07.014

[10] B. Ning, Pairs of Fan-type heavy subgraphs for pancyclicity of 2-connected graphs, Australas. J. Combin. 58 (2014) 127-136.

[11] E.F. Schmeichel and S.L. Hakimi, A cycle structure theorem for Hamiltonian graphs, J. Combin. Theory Ser. B 45 (1988) 99-107. doi:10.1016/0095-8956(88)90058-5

[12] W. Widel, A Fan-type heavy pair of subgraphs for pancyclicity of 2-connected graphs, Discuss. Math. Graph Theory 36 (2016) 173-184.

doi:10.7151/dmgt.1840

Received 20 October 2015

Revised 1 February 2016

Accepted 1 February 2016 